\title{
Distribuição vertical da Anomalocardia flexuosa (Linnaeus, 1767) (Bivalvia: Veneridae) na praia de Mangue Seco (Pernambuco, Brasil)
}

\author{
Severino Adriano de Oliveira Lima ${ }^{1 *}$ \\ Humber Agrelli Andrade ${ }^{2}$ \\ Alfredo Leandro Borie Mojica ${ }^{3}$ \\ Raniere Garcez Costa Sousa ${ }^{1}$ \\ ${ }^{1}$ Universidade Federal de Rondônia, Departamento de Engenharia de Pesca \\ Rua da Paz, 4.376, Lino Alves Teixeira, CEP 76.916-000, Presidente Médici - RO, Brasil \\ ${ }^{2}$ Universidade Federal Rural de Pernambuco, Departamento de Pesca e Aquicultura \\ Rua Dom Manoel de Medeiros, Dois Irmãos, s/n, CEP 52.171-900, Recife - PE, Brasil \\ ${ }^{3}$ Universidade Federal de Alagoas, Departamento de Pesca e Aquicultura \\ Rua Beira Rio s/n, Centro Histórico, CEP 57.200-000, Penedo - AL, Brasil \\ * Autor para correspondência \\ limasao@unir.com
}

Submetido em 26/11/2020

Aceito para publicação em 29/03/2021

\section{Resumo}

O objetivo neste trabalho foi estudar a distribuição vertical da Anomalocardia flexuosa em uma praia arenosa do nordeste brasileiro. Foram obtidas 135 amostras em três meses no ano de 2015. Para testar a hipótese entre as densidades nas profundidades de até $5 \mathrm{~cm}$ (densidade 5) e entre 5 e $10 \mathrm{~cm}$ (densidade 10) foi realizado o teste t-Student. Modelos Lineares Generalizados foram aplicados para estimar a variável resposta densidade 10 com as explicativas espaciais, temporais e de abundância. Os resultados do melhor modelo foram interpolados por métodos geoestatísticos. As densidades foram significativamente maiores na densidade $5\left(450\right.$ ind. $\left.\mathrm{m}^{2}\right)$ do que na densidade 10 (7 ind. $\left.\mathrm{m}^{2}\right)$. Entre os modelos avaliados, o com Binomial Negativa inflada de zeros foi o selecionado, sendo as predições interpoladas verossímeis com o observado, e as variáveis utilizadas foram: densidade 5, latitude, longitude (transformada) e mês (contínuo). A ocupação de A. flexuosa em camadas mais profundas do substrato pode decorrer de um enterramento acidental e ser atribuída a forçantes físicas, e não ser uma causa comportamental. Dessa maneira, o manejo sobre essa espécie deve considerar meios de captura que não penetrem o substrato além da camada preferencial, para assim não impactar outros organismos não alvo da pesca.

Palavras-chave: Berbigão; Enterramento; Macrofauna; Praia arenosa

\section{Abstract}

Vertical distribution of Anomalocardia flexuosa (Linnaeus, 1767) (Bivalvia: Veneridae) on the beach of Mangue Seco (Pernambuco, Brazil). The objective of this work was to study the vertical distribution of Anomalocardia flexuosa on a sandy beach in Northeast Brazil. A total of 135 samples were obtained during three 
months in 2015. The hypothesis between densities at depths up to $5 \mathrm{~cm}$ (density 5) and between 5 and $10 \mathrm{~cm}$ (density 10) was evaluated with the Student t-test. Generalized linear models were applied to evaluate the variable density 10 with spatial, temporal and abundance explanations. The results of the best model were interpolated by geostatistical methods. Densities were significantly higher at density 5 (450 ind. $\left.\mathrm{m}^{2}\right)$ than at density 10 (7 ind. $\mathrm{m}^{2}$ ). Among the models evaluated, the zero-inflated negative binomial was selected, with the interpolated predictions being credible considering that observed, and the variables used were density 5 , latitude, longitude (transformed) and month (continuous). The presence of A. flexuosa in deeper layers of the substrate may result from an accidental burial and may be attributed to physical forcing and not to a behavioral cause. Therefore, the management of this species must consider fishing gear that does not penetrate the substrate beyond the preferred layer, thus not impacting non-target animals in fishing.

Key words: Berbigão; Burial; Macrofauna; Sandy beach

\section{Introdução}

Os macroinvertebrados bentônicos são grupos de organismos que apresentam relação direta com os substratos que ocupam (DAY et al., 1989). Utilizados como bioindicadores ambientais, desempenham processos ecológicos de grande importância, como fluxos de energia e ciclagem de nutrientes (GASTON et al., 1998; HERMAN et al., 1999; YSEBAERT et al., 2002). Nas praias arenosas, esse grupo é composto por espécies permanentes que normalmente têm distribuições agregadas (BROWN; MCLANCHLAN, 1990), sendo o filo Mollusca um dos mais difundidos neste tipo de ambiente (RUEDA et al., 2009). Várias espécies de moluscos são exploradas comercialmente nas praias arenosas, com destaque para os bivalves (SILVA-CAVALCANTI; COSTA, 2011; BELÉM et al., 2013; LIMA et al., 2020).

A distribuição dos moluscos bivalves nos mais diversos habitat, incluindo praias arenosas, está condicionada a interações bióticas, e estas podem ter maior relevância que as forças físicas (CORTE et al., 2014). A intraespecífica competição e a localização podem controlar a dinâmica populacional de bivalves em um ecossistema (DE FOUW et al., 2020). Assim, sugere-se que fatores ecológicos e físicos devem ser considerados concomitantemente para melhor entender as variações da endofauna bentônica (DEFEO; MARTÍNEZ, 2003; CORTE et al., 2014).

Entre as variáveis abióticas, a profundidade é uma das mais importantes para o desenvolvimento dos bivalves (GASPAR et al., 2002). O aumento da profundidade de enterramento é uma estratégia contra predadores forrageadores (PIERSMA et al., 1994; ZAKLAN; YDENBERG, 1997), protegendo os bivalves de serem carreados por correntezas e contra temperatura extremas (RATCLIFFE et al., 1981; SUTHERLAND, 1982). Porém, o enterramento interfere também na alimentação, sendo a faixa de profundidade um fator primordial para atender às necessidades de energia dentro de um certo tempo de imersão (DE GOEIJ; HONKOOP, 2002).

A profundidade de enterramento pode ser um fator limitante, principalmente para bivalves que apresentam comprimento e sifão curtos (HADDON et al., 1987; ZWARTS; WANINK, 1989), como, por exemplo, grande parte das espécies da família Veneridae (ANSELL, 1961), à qual Anomalocardia flexuosa pertence. Essa espécie é um dos bivalves marinhos mais explorados na costa brasileira (SILVA-CAVALCANTI; COSTA, 2011), especialmente na praia de Mangue Seco, em Pernambuco, principal região produtora no Brasil e no mundo. Nas últimas estimativas estatísticas disponíveis no Brasil, com detalhes por estado, o registro máximo de captura ocorreu em 2007 (4.716 toneladas) no estado de Pernambuco, sendo que no Brasil, no mesmo ano, foi estimada uma captura de aproximadamente 13.710 toneladas de moluscos marinhos (IBAMA, 2009).

A exploração de $A$. flexuosa é praticamente toda realizada no Brasil, mas a espécie ocorre nas Antilhas e na costa leste das Américas (ABBOTT, 1974). Assim como outros bivalves, essa espécie se estabelece em variados habitat e tem como característica apresentar distribuição espacial agregada, o que pode acarretar amostragens com ausência de indivíduos. Na ecologia de populações, os dados com contagem zero de indivíduos 
nas amostras são comuns devido às restrições ecológicas dos sistemas biológicos (BLASCO-MORENO et al., 2019). Portanto, um modelo de distribuição de probabilidade contínua pode não ser o ideal para avaliação de distribuição agregada, pois frequentemente esses dados com ausências também exibem uma variação maior que a média, fenômeno conhecido como super dispersão, sendo que os modelos com zeros inflados podem ser uma alternativa (HEILBRON, 1994). Esse tipo de abordagem também é efetuado por modelos de Hurdle (MULLAHY, 1986) e estes permitem a obtenção de estimativas confiáveis para distribuições biológicas dos bivalves. Portanto, o objetivo neste trabalho foi estudar o perfil de distribuição vertical de A. flexuosa em uma praia de solo arenoso.

\section{Material e Métodos}

\section{Área de estudo}

A praia de Mangue Seco está localizada no município de Igarassu - Pernambuco, nordeste do Brasil (Figura 1), tendo como principais atividades o turismo e a extração do molusco bivalve Anomalocardia flexuosa, que ocorre manualmente ou com auxílio de petrechos que revolvem o sedimento (RODRIGUES et al., 2013; LIMA et al., 2020). O banco da praia é predominantemente arenoso, com altos percentuais de areia grossa e fina, e se estende no sentido Sul-Norte, desde a foz do Rio Timbó até a foz Sul do Canal de Santa Cruz, abrangendo uma área de aproximadamente 2,7 km² (LAVANDER et al., 2011; LIMA; ANDRADE, 2018).

\section{Coleta dos dados}

Foram obtidas 135 amostras em três meses de coletas, sendo 47 no mês de julho, 61 em agosto e 27 em setembro no ano de 2015 (Figura 1). As amostras foram realizadas em pontos aleatórios ao longo de todo o banco de praia, com o emprego de um coletor cilíndrico de policloreto de polivinila (PVC) de $19 \mathrm{~cm}$ de diâmetro, que foi enterrado até $10 \mathrm{~cm}$ de profundidade. O material coletado foi dividido em dois estratos de profundidade, sendo o primeiro na superfície até $5 \mathrm{~cm}$ (profundidade 5) e o segundo em camada inferior, de 5 a $10 \mathrm{~cm}$ (profundidade 10). O sedimento e os exemplares de $A$.

FIGURA 1: Praia de Mangue Seco localizada no município de Igarassu, estado de Pernambuco, Nordeste do Brasil. Círculos abertos, triângulos abertos e quadrados fechados representam pontos de coletas nos meses de julho, agosto e setembro de 2015 , respectivamente.

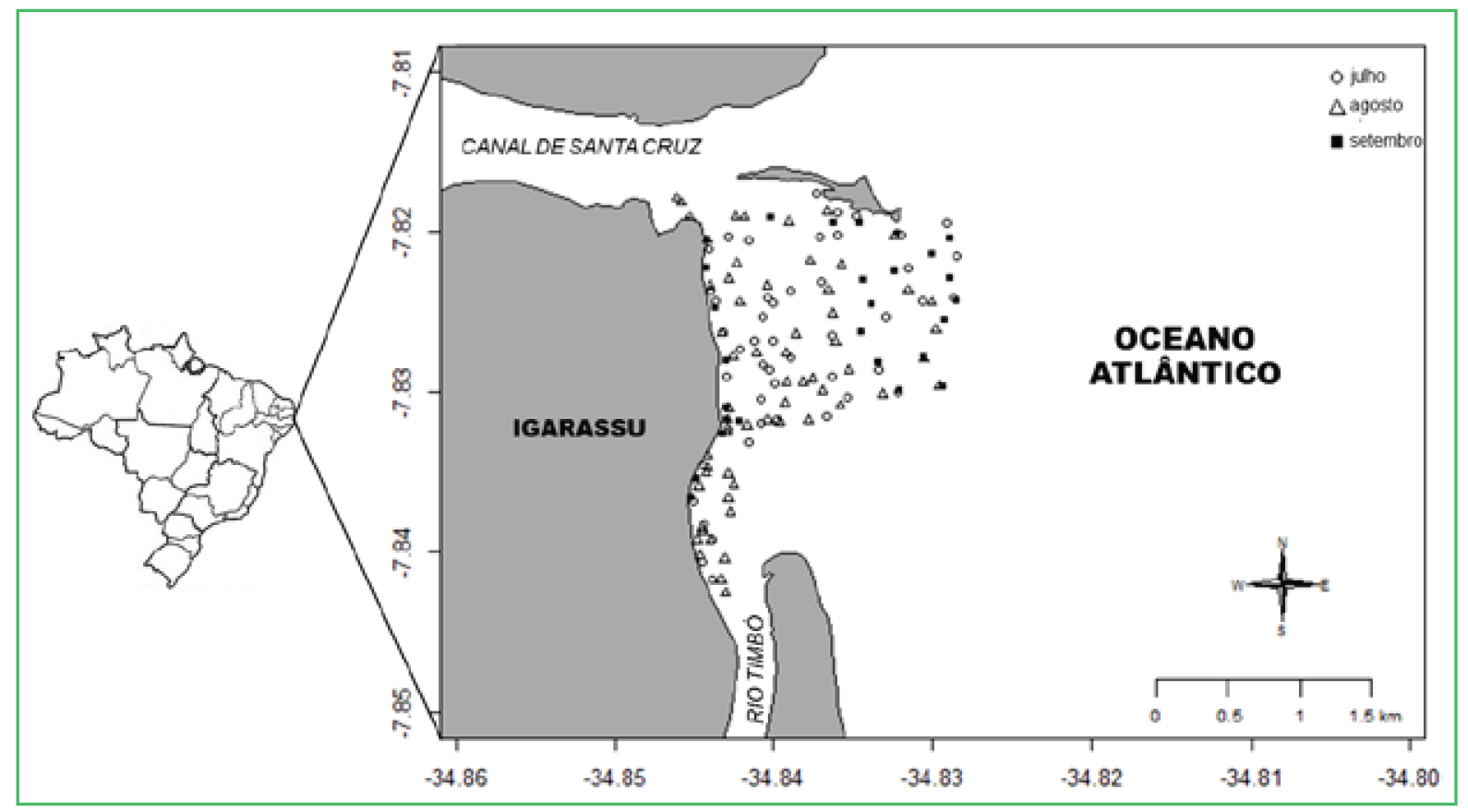


flexuosa foram lavados juntos e peneirados em malha quadrada de $1 \mathrm{~mm}$ entre nós adjacentes, etapa seguida pela contabilização, já em laboratório.

\section{Análise dos dados}

Para testar a hipótese entre as densidades de A. flexuosa na profundidade de até $5 \mathrm{~cm}$ (P5) e as densidades na profundidade entre 5 e $10 \mathrm{~cm}$ (P10), foi realizado o teste t-Student $(\alpha=0,05)$. Modelos Lineares Generalizados (GLMs) foram aplicados para avaliar a variável resposta densidade 10, considerando Poisson, Hurdle Poisson (HP), Poisson inflada de zeros (ZIP) e Binomial Negativa inflada de zeros (ZINB) (Tabela 1). A seleção do melhor modelo foi feita com base no Critério de Informação de Akaike (AIC) (AKAIKE, 1973), Critério de Informação Bayesiano (BIC) (SCHWARZ, 1978), logaritmo da probabilidade (logLik) e número de zeros previstos (Zero), além do teste de Vuong ( $\alpha=$ 0,05) (VUONG, 1989).

As variáveis explicativas foram P5, a latitude, a longitude e a transformação da latitude \{log [(latitude $+34.1) / 10]\}$ e da longitude [(longitude +43.8$) / 10]$ (COELHO et al., 2020). Espacialmente também foi considerada a estratificação da área realizada, de acordo com Lima e Andrade (2018), em que foram considerados os efeitos de bordas do banco nos contatos com os corpos de água circundantes e a proximidade da linha de praia. Além disso, foi analisado o padrão temporal, sendo os meses testados como fator ou como variável contínua.

Os resultados das densidades observadas para $A$. flexuosa na profundidade entre 5 e $10 \mathrm{~cm}$, assim como os preditos pelo melhor modelo, foram interpolados por métodos geoestatísticos, utilizando cálculos com o inverso da distância. Todas as análises foram realizadas com o programa R 4.0.0 (R CORE TEAM, 2020), e cálculos geoestatísticos através do pacote gstat (PEBESMA; GRAELER, 2017).

\section{Resultados}

As densidades de $A$. flexuosa foram significativamente maiores $(t=7,78 ; d f=134 ; p=$ $1,69 \times 10^{-16}$ ) na P5, com média de 450 ind. $\mathrm{m}^{2}$ (661 ind. $\mathrm{m}^{2}$ ), enquanto na P10 a média foi de 7 ind. $\mathrm{m}^{2}$ (24 ind. $\mathrm{m}^{2}$ ) (Figura 2). Nas coletas efetuadas na camada mais profunda (P10), aproximadamente $91 \%$ das amostras tiveram densidade zero.

TABELA 1: Modelos de probabilidade de Poisson, Hurdle Poisson, Poisson inflada de zeros e Binomial Negativa inflada de zeros para as densidades da Anomalocardia flexuosa nas profundidades entre 5 e $10 \mathrm{~cm}$.

\begin{tabular}{|c|c|c|c|c|}
\hline Modelo & \multicolumn{3}{|c|}{ Função da probabilidade } & \\
\hline Poisson & & $P\left(Y_{i}=y_{i}\right)=\frac{e^{-\mu_{i}} \mu_{i}^{Y}}{y_{i} !}, \quad y_{i} \geq$ & & \\
\hline Hurdle Poisson & & $\left.=y_{i}\right)=\left\{\begin{array}{l}\left(1-p_{i}\right) \frac{e^{-\mu_{i}} \mu_{i}^{y_{i}}}{\Gamma\left(1-e^{-\mu_{i}}\right) y_{i} !} \\
{ }^{\prime}\end{array}\right.$ & $\begin{array}{l}y_{i}=\mathrm{C} \\
y_{4}>\mathrm{C}\end{array}$ & \\
\hline $\begin{array}{l}\text { Poisson } \\
\text { Inflada de zeros }\end{array}$ & & $\left.Y_{i}=y_{i}\right)=\left\{\begin{array}{l}p_{i}+\left(1-p_{i}\right) e^{-\mu} \\
\left(1-p_{i}\right) \frac{e^{-\mu_{i}} \mu_{i}^{y_{i}}}{y_{i} !}\end{array}\right.$ & $\begin{array}{l}y_{i}=0 \\
y_{i}>0\end{array}$ & \\
\hline $\begin{array}{l}\text { Binomial } \\
\text { Negativa } \\
\text { Inflada de zeros }\end{array}$ & $P\left(Y_{i}=y_{i}\right)=$ & $\begin{array}{r}p_{i}+\left(1-p_{i}\right) \\
\left(1-p_{i}\right) \frac{\Gamma\left(y_{i}+\emptyset\right)}{\Gamma(\emptyset) \cdot y_{i} !}\left(\frac{\emptyset}{\mu_{i}+\emptyset}\right)^{\emptyset}(1-\end{array}$ & $\begin{array}{l}\left.\frac{\varphi}{+\theta}\right)^{0} \\
\left(\frac{\theta}{+\phi}\right)^{3 \pi}\end{array}$ & $\begin{array}{l}y_{i}=\mathrm{C} \\
y_{i}>\mathrm{C}\end{array}$ \\
\hline
\end{tabular}


FIGURA 2: Barras com as médias e linhas com os desvios padrão das densidades de Anomalocardia flexuosa na praia de Mangue Seco.

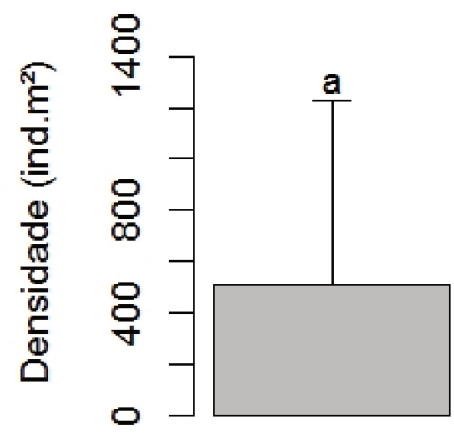

5 b

10

\section{Profundidade $(\mathrm{cm})$}

$\mathrm{Na}$ análise exploratória, as tendências entre a variável resposta $\mathrm{P} 10$ e as variáveis explicativas são apresentadas na Figura 3. A variável resposta cresceu a partir das densidades superiores a 1.000 ind $\mathrm{m}^{2}$ na $\mathrm{P} 5$, atingindo um pico com aproximadamente 2.500 ind. $\mathrm{m}^{2}$, e decresceu após esse valor (Figura $3 \mathrm{~A}$ ). Nas variáveis espaciais de latitude e latitude transformada (Figura $3 \mathrm{~B} \mathrm{e} \mathrm{C}$ ) e longitude e longitude transformada (Figura $3 \mathrm{D}$ e E), a variável resposta oscilou com dois picos em valores mais baixos. Considerando os meses como fatores, ocorreram maiores valores no mês de julho, agosto e setembro (Figura $3 \mathrm{H}$ ), respectivamente, ficando evidente um decréscimo quando os meses foram tratados como variável contínua (Figura 3 I).

As variáveis selecionadas para modelos mais parcimoniosos foram $\mathrm{P} 5$, latitude, longitude transformada (Longitude.t) e mês (variável contínua). Entre os quatro modelos avaliados, o ZINB foi o que apresentou os melhores indicadores, exceto para o número de zeros preditos (Tabela 2). A superioridade do ajuste do modelo ZINB foi significativa quando comparada a todos

FIGURA 3: Gráficos exploratórios dos padrões entre a variável resposta profundidade entre 5 e $10 \mathrm{~cm}(\mathrm{P} 10)$ com as variáveis explicativas: Densidade de Anomalocardia flexuosa na profundidade até $5 \mathrm{~cm}$ (P5) (A); Latitude (B); Latitude transformada - Latitude.t (C); Longitude (D); Longitude transformada - Longitude.t (E); Mês como fator (F); Mês como variável contínua (G).

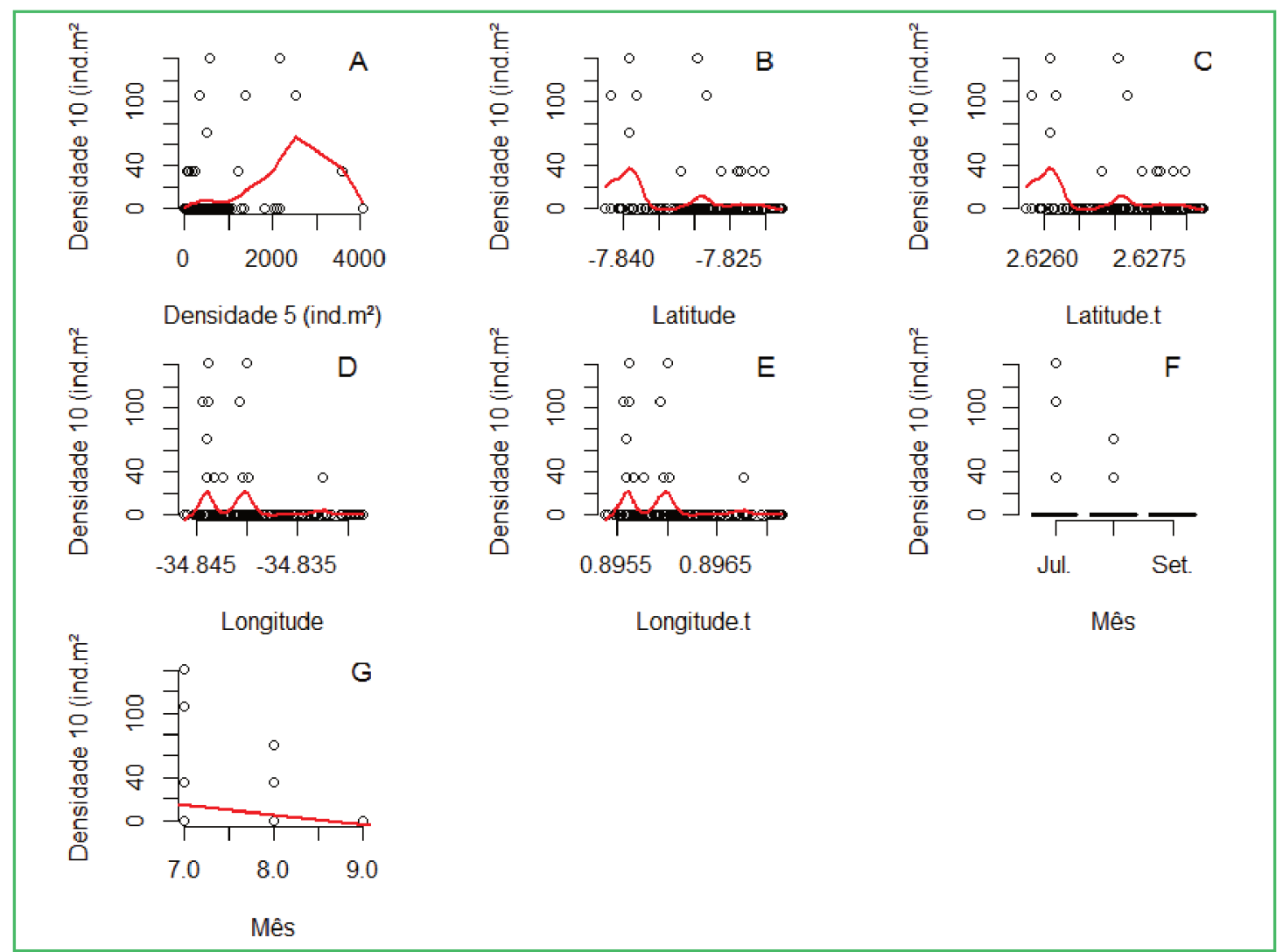


os outros modelos como, por exemplo, ao segundo melhor, que foi o ZIP, comprovada pelo teste de Vuong $(\mathrm{V}=-1,99, \mathrm{p}=0,02)$ para estimativas de AIC e BIC.

As estimativas do modelo ZINB foram significativas para latitude e log (theta) nos dados com contagem superior a zero, enquanto nas condições de densidade zero, P5 e mês foram significativos (Tabela 3).

TABELA 2: Comparativo dos quatro modelos avaliados com indicadores do critério de informação de Akaike (AIC), critério de informação bayesiano (BIC), logaritmo da verossimilhança ( $\log L i k)$ e número de zeros preditos.

\begin{tabular}{lcccc}
\hline \multirow{2}{*}{ Indicadores } & \multicolumn{4}{c}{ Estrutura do modelo } \\
\cline { 2 - 5 } & Poisson & $\begin{array}{c}\text { Hurdle } \\
\text { Poisson }\end{array}$ & ZIP & ZINB \\
\hline AIC & 2461 & 287 & 280 & 191 \\
BIC & 2476 & 316 & 310 & 239 \\
logLik & -1226 & -133 & -130 & -83 \\
Zero & 51 & 55 & 54 & 54 \\
\hline
\end{tabular}

As estimativas dos parâmetros podem ser usadas para calcular predições que correspondem às médias das densidades de $A$. flexuosa em função das diferentes variáveis explicativas (Figura 4). O efeito da densidade na P5 foi crescente principalmente a partir de 3.000 ind. $\mathrm{m}^{2}$ (Figura $4 \mathrm{~A}$ ). A densidade tendeu a decrescer na latitude (Figura $4 \mathrm{~B}$ ), principalmente após $-7,845^{\circ} \mathrm{S}$, e também de maneira suave para maiores valores da longitude transformada (Figura $4 \mathrm{C}$ ). Nos meses, a variável resposta foi decrescente, com queda intensa no mês de setembro (Figura 4 D).

As interpolações das densidades de A, flexuosa na P10 foram plotadas nos painéis superiores, já as predições do modelo são demonstradas nos painéis inferiores (Figura 5). As densidades foram maiores nos meses de julho (Figura $5 \mathrm{~A}$ ) e agosto (Figura $5 \mathrm{~B}$ ), respectivamente, enquanto no mês de setembro (Figura 5 C) não ocorreram indivíduos. De maneira geral, nos meses em que o bivalve ocorreu nas amostragens, as densidades foram maiores em latitudes e longitudes mais baixas. As predições seguiram padrões similares com as densidades observadas para os meses de julho (Figura 5 D), agosto (Figura 5 E) e setembro (Figura $5 \mathrm{~F}$ ), porém com estimativas de densidades menores nos meses de presença de A. flexuosa.

TABELA 3: Estimativas dos coeficientes dos parâmetros do modelo negativo binomial inflado de zero, selecionado para predições da variável resposta densidade da Anomalocardia flexuosa entre as profundidades 5 e $10 \mathrm{~cm}(\mathrm{P} 10)$.

\begin{tabular}{lcccc}
\hline & \multicolumn{3}{c}{ Coeficientes do modelo de contagem (binomial negativa com ligação log) } \\
\cline { 2 - 5 } & Estimativa & Erro padrão & Valor de $\mathbf{z}$ & $\operatorname{Pr}(>|\mathbf{z}|)$ \\
\hline Intercepto & $-856,8272$ & 517,9831 & $-1,6542$ & 0,0981 \\
P5 & 0,0001 & 0,0001 & 0,8144 & 0,4154 \\
Latitude & $-62,9818$ & 19,8867 & $-3,1670$ & 0,0015 \\
Longitude,t & 413,0798 & 463,7496 & 0,8907 & 0,3731 \\
Mês & $-0,3187$ & 0,2986 & $-1,0671$ & 0,2859 \\
log(theta) & 2,0329 & 0,5526 & 3,6789 & 0,0002 \\
\hline & $\mathbf{C o e f i c i e n t e ~ d o ~ m o d e l o ~ c o m ~ z e r o ~ i n f l a d o s ~ ( b i n o m i a l ~ c o m ~ l i g a c ̧ a ̃ o ~ l i n k ) ~}$ \\
\hline Intercepto & Estimativa & Erro padrão & Valor de $\mathbf{z}$ & $\operatorname{Pr}(>|\mathbf{z}|)$ \\
Densidade 5 & $-1021,3994$ & 1440,3476 & $-0,7091$ & 0,4782 \\
Latitude & $-0,0009$ & 0,0004 & $-1,9627$ & 0,0497 \\
Longitude,t & 60,7661 & 62,0503 & 0,9793 & 0,3274 \\
Mês & 1657,7823 & 1291,4567 & 1,2837 & 0,1993 \\
\hline
\end{tabular}


FIGURA 4: Predições do modelo Binomial Negativa inflada de zeros, para as variáveis explicativas: Densidade de Anomalocardia flexuosa na profundidade até $5 \mathrm{~cm}$ (P5) (A); Latitude (B); Longitude transformada - Longitude.t (C); Mês como variável contínua (D).

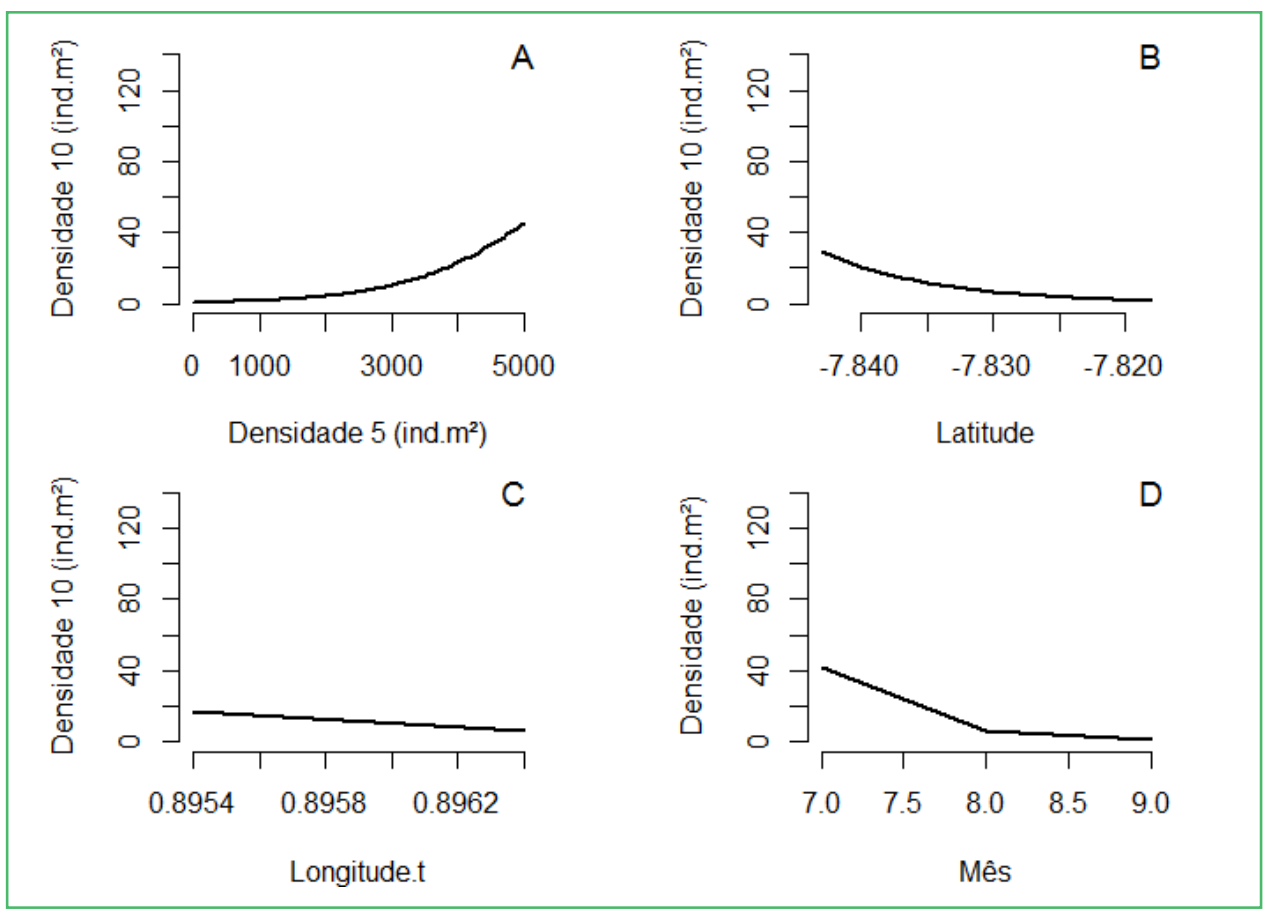

FIGURA 5: Interpolações (inverso da distância) das densidades de Anomalocardia flexuosa entre as profundidades 5 e $10 \mathrm{~cm}$ (P10) na praia de Mangue. Painéis superiores são os resultados dos observados nos meses de julho (A), agosto (B) e setembro (C). Painéis inferiores são os resultados das predições do modelo nos meses de julho (D), agosto (E) e setembro (F).

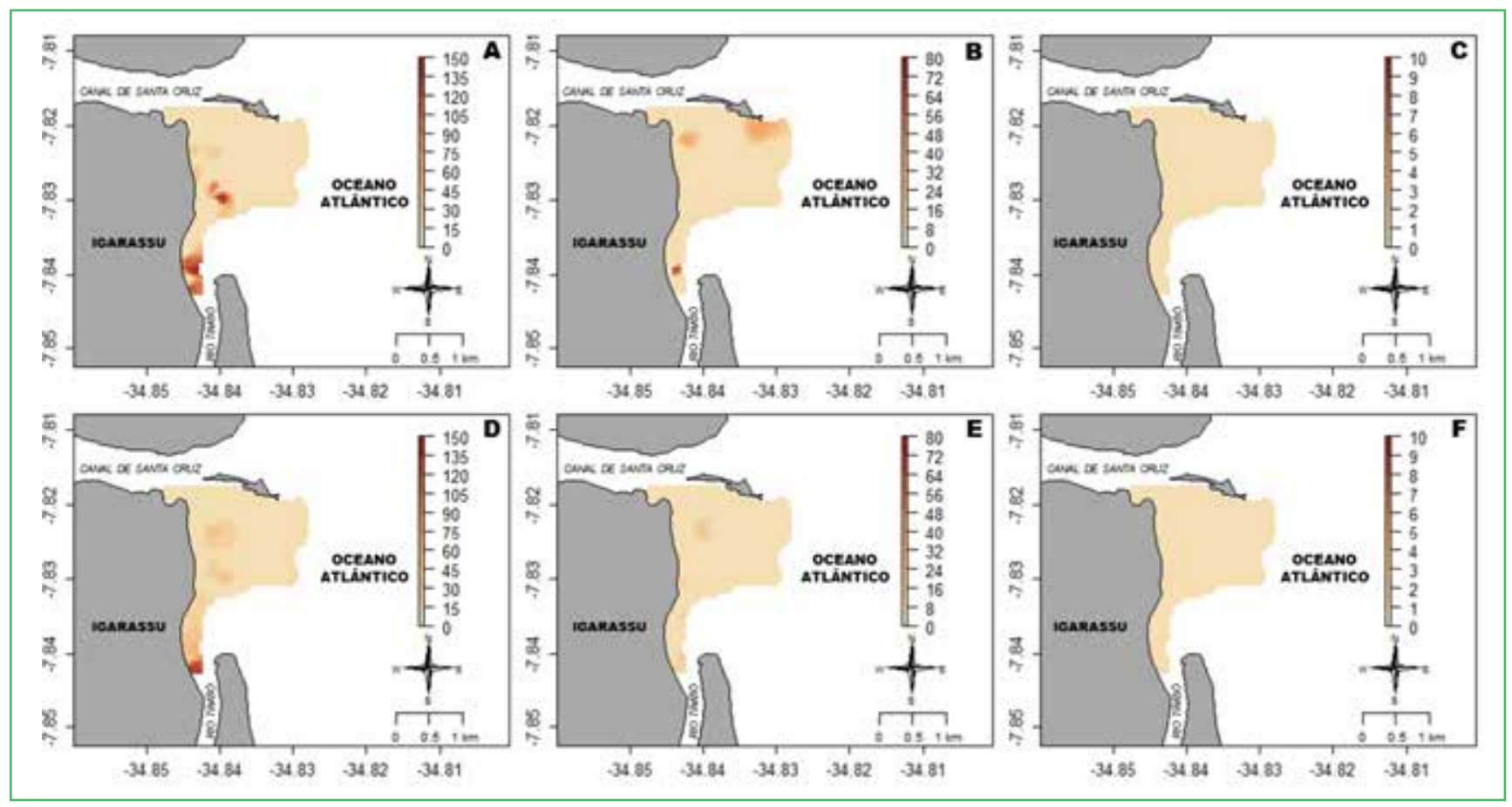




\section{Discussão}

As densidades nas amostras do estrato mais profundo (P10) apresentaram muitos valores zero, o que pode estar relacionado às características morfológicas de $A$. flexuosa, principalmente pelo sifão, que é curto e primitivo (ANSELL, 1961; NARCHI, 1972), mesmo comparado a uma outra espécie de venerídeo (Protothaca pectorina) que também apresenta esta estrutura pouco desenvolvida (GUERÓN; NARCHI, 2000). O sifão é determinante na distribuição vertical de bivalves (ZWARTS; WANINK, 1989). Densidades elevadas abaixo dos $10 \mathrm{~cm}$ da superfície da areia são pouco prováveis para $A$. flexuosa, sendo a camada mais superficial (P5) a que mais apresenta exemplares competindo por espaço e alimento (NARCHI, 1974; FERREIRA et al., 2015). Agentes causadores de estresse e competição, como, por exemplo, locais com ressuspensão frequente de sedimentos (NARCHI, 1974), podem ter condicionado a esse bivalve ficar imerso em zonas mais profundas do sedimento. Portanto, os valores de zeros decorrentes da distribuição de A. flexuosa no estrato de maior profundidade não foram aleatórios, sendo estes estruturais, corroborando o uso de modelos inflados (BLASCO-MORENO et al., 2019), pois mesmo sem considerar a estratificação por profundidade, a distribuição de Poisson pode não se ajustar bem a dados de densidade de macroinvertebrados (GRAY, 2005).

Nas variáveis respostas testadas neste trabalho, as medidas espaciais selecionadas foram a latitude e a longitude transformada, sendo os resultados melhores do que o zoneamento da área proposto por Lima e Andrade (2018). Porém, ressalta-se que na proposta do trabalho desses autores, a distribuição de $A$. flexuosa foi avaliada horizontalmente com o core (amostrador), sem separação de estratos de profundidade e com amostragem até 10 $\mathrm{cm}$, assim o delineamento pode ser eficiente quando considerado o agregado, mas não para indivíduos presentes somente na profundidade maior do que $5 \mathrm{~cm}$. Nas variáveis temporais que foram tratadas de duas formas (contínua ou fator), a seleção da contínua pode ter decorrido pelo número baixo de meses coletados, implicando um decaimento linear.

No modelo selecionado (ZINB), a maioria das variáveis explicativas apresentaram tendências negativas, assim ocorreu que, com o aumento no valor dessas variáveis, as densidades na P10 diminuiriam. No entanto, a densidade no estrato mais superficial (P5) apresentou padrão oposto, o que possivelmente é consequência de uma competição intraespecífica por espaço e alimento, pois indivíduos adultos de A. flexuosa podem inibir a presença de pequenos (BOEHS et al., 2008). Além disso, maiores densidades na parte superior devem proporcionar tensão física, levando alguns desses exemplares, que já estejam com pouca latência, para o setor mais profundo, apesar da capacidade de mobilidade horizontal deste bivalve (NARCHI, 1972).

Nas estimativas das variáveis explicativas espaciais, as predições considerando a latitude tiveram densidades maiores nas proximidades do Rio Timbó. Porém, em densidades agregadas, tanto essa região quanto na saída do Canal de Santa Cruz apresentaram densidades intermediárias para o banco extrativista avaliado (LIMA; ANDRADE, 2018). Assim, a presença do bivalve na P10 somente em latitudes mais baixas pode ser um processo passivo condicionado ao maior fluxo e força da correnteza, que revolvem o sedimento e enterram mais os indivíduos na saída do Rio Timbó e não propriamente decorrente da ação da espécie. Na morfodinâmica de praias arenosas, os atributos físico-ambientais afetam o estabelecimento e a distribuição de várias espécies, pois praias com características dissipativas com areias finas, baixa penetração e alto teor de água no substrato e larga zona de arrebentação (que facilita a dissipação da energia das ondas) são mais favoráveis para as espécies quando comparados aos atributos das praias reflexivas, que têm propriedades contrárias (SHORT, 1996; DEFEO et al., 2001). Há grande influência de três gradientes ambientais físicos na distribuição das espécies, o espraiamento (frequência e velocidade), a granulometria e a dinâmica da erosão e da deposição do sedimento, fatores que sugerem a redução de espécies quando aumentam em direção ao extremo reflexivo, formando assim a hipótese de severidade ambiental multicausal (BRAZEIRO, 2001). Porém, não é totalmente ausente a presença de A. flexuosa nas latitudes mais baixas e longitudes mais próximas à costa em estratos mais profundos.

As estimativas dos maiores valores de densidades na P10 ocorreram no mês de julho. Esse mês coincide 
com o maior índice pluviométrico em relação aos demais meses amostrados, registrado para o local de estudo pela Agência Pernambucana de Águas e Clima (Apac). A pluviosidade intensa provoca redução no número de indivíduos de A. flexuosa (BELÉM et al., 2013; OLIVEIRA et al., 2014), porém para o estrato mais profundo foi um fator favorável. Há duas possibilidades para a ocorrência desse fenômeno, a primeira é que a chuva provoca um maior revolvimento no sedimento, levando os bivalves mais para o fundo, e a segunda pode estar relacionada à mortalidade da espécie, pois a latência neste período diminui e os indivíduos ficam mais propensos a serem enterrados, entretanto essas conjecturas devem ser avaliadas em estudos posteriores.

As predições do modelo interpoladas foram verossímeis com o observado, contudo com tendência de valores mais baixos, principalmente nos meses de julho e agosto. No entanto, as variáveis utilizadas no modelo demonstraram uma pequena parte da variabilidade, sendo as que tiveram mais preponderância foram a latitude e o mês, que podem ter ocorrido em virtude da movimentação imposta por correntes de saídas de rio e pluviosidade, respectivamente. Portanto, a ocupação de A. flexuosa em camadas mais profundas do substrato pode decorrer de um enterramento acidental e ser atribuída a forçantes físicas, e não ser uma causa comportamental do bivalve. Assim, o manejo dessa espécie, que é economicamente importante, deve também considerar meios de captura que não penetrem o substrato além da camada preferencial, e, dessa maneira, não impactar outros organismos não alvo da pesca.

\section{Agradecimentos}

À Coordenação de Aperfeiçoamento de Pessoal de Nível Superior (CAPES), por conceder uma bolsa de estudo de pós-graduação no momento da pesquisa ao primeiro autor.

\section{Referências}

ABBOTT, R. T. American seashell. New York: Van Nostrand, 1974. $663 \mathrm{p}$.

AKAIKE, H. Information theory and an extension of the maximum likelihood principle B.N. In: PETROVAND, F. C. (Ed.), The
Second Symposium on Information Theory. Budapest: Akademial Kiado, 1973. p. 267-281.

ANSELL, A. O. The functional morphology of the British species of Veneracea (Eulamellibranchia). Journal of the Marine Biological Association of the United Kingdom, Cambridge, v. 41, n. 2, p. 489-517, 1961.

BELÉM, T. P.; MOURA, R. S. T.; HENRY-SILVA, G. G. Distribuição e densidade do bivalve Anomalocardia brasiliana em praias do Rio Grande do Norte durante um período de pluviosidade atípica. Biotemas, Florianópolis, v. 26, n. 1, p. 109-122, 2013.

BLASCO-MORENO, A.; PÉREZ-CASANY, M.; PUIG, P.; MORANTE, M.; CASTELLS E. What does a zero mean? Understanding false, random and structural zeros in ecology. Methods in Ecology and Evolution, London, v. 10, n. 7, p. 949959, 2019.

BOEHS, G.; ABSHER, T. M.; CRUZ-KALED, A. C. Ecologia populacional de Anomalocardia brasiliana (Gmelin, 1791) (Bivalvia, Veneridae) na Baía de Paranaguá, Paraná, Brasil. Boletim do Instituto de Pesca, São Paulo, v. 34, n. 2, p. 259-270, 2008.

BRAZEIRO, A. Relationship between species richness and morphodynamics in sandy beaches: what are the underlying factors? Marine Ecology Progress Series, Luhe, v. 224, p. 35-44, 2001.

BROWN, A. C.; MCLACHLAN, A. Ecology of sandy shores. Amsterdam: Elsevier, 1990. 327 p.

COELHO, R.; INFANTE, P.; SANTOS, M. N. Comparing GLM, GLMM, and GEE modeling approaches for catch rates of bycatch species: A case study of blue shark fisheries in the South Atlantic. Fisheries Oceanography, Cambridge, v. 29, n. 2, p. 169-184, 2020. CORTE, G. N.; YOKOYAMA, L. Q.; AMARAL, A. C. Z. An attempt to extend the Habitat Harshness Hypothesis to tidal flats: a case study of Anomalocardia brasiliana (Bivalvia: Veneridae) reproductive biology. Estuarine, Coastal and Shelf Science, New York, v. 150, v. 15, p. 136-141, 2014.

DAY, J. W.; HALL, C. A. S.; KEMP W. M.; YÁNEZ-ARANCIBIA A. Estuarine Ecology. New York: Wiley-Interscience Publication, 1989. $576 \mathrm{p}$.

DE FOUW, J.; VAN DER ZEE, E. M.; VAN GILS, J.A.; ERIKSSON, B. K.; WEERMAN, E. J.; DONADI, S.; VAN DER VEER, H. W.; OLFF, H.; PIERSMA, T.; VAN DER HEIDE, T. The interactive role of predation, competition and habitat conditions in structuring an intertidal bivalve population. Journal of Experimental Marine Biology and Ecology, Amsterdam, v. 523, 151267, 2020.

DE GOEIJ, P.; HONKOOP, P. J. C. The effect of immersion time on burying depth of the bivalve Macoma balthica (Tellinidae). Journal of Sea Research, Amsterdam, v. 47, n. 2, p. 109-119, 2002.

DEFEO, O.; GÓMEZ, J.; LERCARI, D. Testing the swash exclusion hypothesis in sandy beach populations: the mole crab Emerita brasiliensis in Uruguay. Marine Ecology Progress Series, Luhe, v. 212, p. 159-170, 2001.

DEFEO, O.; MARTÍNEZ, G. The habitat harshness hypothesis revisited: life history of the isopod Excirolana braziliensis in sandy beaches with contrasting morphodynamics. Journal of the Marine Biological Association of the United Kingdom, Cambridge, v. 83, n. 2, p. 331-340, 2003.

FERREIRA, A. J.; NETO, R. L. B.; KOLM, H. E.; ABSHER, T. M. Relationship between reproductive cycle of Anomalocardia 
brasiliana (Mollusca: Veneridae) and the suspended particulate matter in the Paranagua Estuarine Complex, Brazil. Pan-American Journal of Aquatic Sciences, Rio Grande, v. 10, n. 1, p. 44-54, 2015.

GASPAR, M. B.; SANTOS, M. N.; VASCONCELOS, P.; MONTEIRO, C. C. Shell morphometric relationships of the most common bivalve species (Mollusca: Bivalvia) of the Algarve coast (southern Portugal). Hydrobiologia, Brussels, v. 477, n. 1, p. 7380, 2002.

GASTON, G. R.; RAKOCINSKI, C. F.; BROWN, S. S.; CLEEVELAND C. M. Trophic function in estuaries: response of macrobenthos to natural and contaminant gradients. Marine and Freshwater Research, Collingwood, v. 49, n. 8, p. 833-846, 1998.

GRAY, B. R. Selecting a distributional assumption for modelling relative densities of benthic macroinvertebrates. Ecological Modelling, Amsterdam, v. 185, n. 1, p. 1-12, 2005.

GUERÓN, C. O.; NARCHI, W. Anatomia funcional de Protothaca (Leukoma) pectorina (Lamarck) (Bivalvia: Veneridae). Revista Brasileira de Zoologia, Curitiba, v. 17, n. 4, p. 1007-1039, 2000.

HADDON, M.; WEAR, R. G.; PACKER, H. A. Depth and density of burial by the bivalve Paphies vernticosaas refuges from predation by the crab Ovalipes catharus. Marine Biology, Kiel, v. 94, p. 25-30, 1987.

HEILBRON, D. Zero-altered and other regression models for count data with added zeros. Biometrical Journal, Weinheim, v. 36, n. 5, p. 531-547, 1994.

HERMAN P. M. J.; MIDDELBURG, J. J.; VAN DE KOPPEL, J.; HEIP, C. H. R. Ecology of estuarine macrobenthos. Advances in Ecological Research, Orlando, v. 29, p. 195-240, 1999.

IBAMA. Estatística da pesca 2007 Brasil: grandes regiões e unidades da federação. Brasília: IBAMA, 2009. 175 p.

LAVANDER, H. D.; CARDOSO, L. O.; OLIVEIRA, R. L.; SILVA NETO, S. R.; GÁLVEZ, A. O.; PEIXOTO, S. R. M. 2011. Biologia reprodutiva da Anomalocardia brasiliana (Gmelin, 1791) no litoral norte de Pernambuco, Brasil. Revista Brasileira de Ciências Agrárias, Recife, v. 6, n. 2, p. 344-350, 2011.

LIMA, S. A. O.; ANDRADE, H. A. Zoneamento da área extrativista mais produtiva da Anomalocardia flexuosa (Linnaeus, 1767) (Mollusca: Bivalvia) no mundo. Biotemas, Florianópolis, v. 31, n. 3, p. 33-41, 2018.

LIMA, S. A. O.; ANDRADE, H. A.; GALVEZ, A. O. Selectivity of a fishing gear used in the catch of Anomalocardia flexuosa in the Northeast of Brazil. Ciência Rural, Santa Maria, v. 50, n. 8, 2020.

MULLAHY, J. Specification and testing of some modified count data models. Journal of Econometrics, New York, v. 33, n. 3, p. 341-365, 1986.

NARCHI, W. Comparative study of the functional morphology of Anomalocardia brasiliana (Gmelin, 1791) and Tivela mactroides (Bom, 1778) (Bivalvia: Veneridae). Bulletin of Marine Science, Miami, v. 22, n. 3, p. 643-670, 1972.

NARCHI, W. Aspectos ecológicos e adaptativos de alguns bivalves do litoral paulista. Papéis Avulsos de Zoologia, São Paulo, v. 27, n. 19, p. 235-262, 1974.

OLIVEIRA, I. B.; SILVA NETO, S. R.; LIMA FILHO, J. V. M.; PEIXOTO, S. R. M.; GALVEZ, A. O. Efeito do período chuvoso na extração do molusco bivalve Anomalocardia brasiliana (Gmelin, 1791). Revista Brasileira de Ciências Agrárias, Recife, v. 9, n. 1, p. 139-145, 2014.

PEBESMA, E.; GRAELER, B. gstat - Spatial and SpatioTemporal Geostatistical Modelling, Prediction and Simulation. 2017. Disponível em <https://github.com/edzer/gstat/>.

PIERSMA, T.; VERKUIL, Y.; TULP, I. Resources for longdistance migration of knots Calidris canutus islandica and C. $c$. canutus: how broad is the temporal exploitation of benthic prey in the western and eastern Wadden Sea? Oikos, Copenhagen, v. 71, n. 3, p. 393-407, 1994.

R CORE TEAM. R: a language and environment for statistical computing. 2020. Disponível em <https:/www.R-project.org/>.

RATCLIFFE, P. J.; JONES, N. V.; WALTERS, N. J. The survival of Macoma balthica (L.) in mobile sediments. In: JONES, N.V.; WOLFF, W.J. (Ed.), Feeding and survival strategies estuarine organisms. New York: Plenum Press, 1981. p. 91-108.

RODRIGUES, S.; LAVANDER, H.; OLIVEIRA, L.; BATISTA, A.; OLIVEIRA, I.; GÁLVEZ, A. O. Distribuição e abundância relativa do berbigão, Anomalocardia brasiliana, na praia de Mangue Seco, Pernambuco, Brasil. Arquivos de Ciências do Mar, Fortaleza, v. 46, n. 2, p. 70-75, 2013.

RUEDA, J.; GOFAS, S.; URRA, J.; SALAS, C. A highly diverse molluscan assemblage associated with eelgrass beds (Zostera marina L.) in the Alboran Sea: microhabitat preference, feeding guilds and biogeographical distribution. Scientia Marina, Barcelona, v. 73, n. 4, p. 679-700, 2009.

SCHWARZ, G. Estimating the dimension of a model. Annals of Statistics, Philadelphia, v. 6, n. 2, p. 461-464, 1978.

SHORT, A. D. The role of wave height, period, slope, tide range and embaymentisation in beach classifications: a review. Revista Chilena de Historia Natural, Santiago, v. 69, p. 589-604, 1996.

SILVA-CAVALCANTI, J. S.; COSTA, M. F. Fisheries of Anomalocardia brasiliana in Tropical Estuaries. Pan-American Journal of Aquatic Sciences, Rio Grande, v. 6, n. 2, p. 86-99, 2011. SUTHERLAND, W. J. Spatial variation in the predation of cockles by oystercatchers at Traeth Melynog, Anglesey. I. The cockle population. Journal of Animal Ecology, London, v. 51, n. 2, p. 481-489, 1982.

VUONG, Q. H. Likelihoood ratio tests for model selection and non-nested hypotheses. Econometrica, New Haven, v. 57, n. 2, p. 307-333, 1989.

YSEBAERT, T.; MEIRE, P.; HERMAN, P. M. J.; VERBEEK H. Macrobenthic species response surfaces along estuarine gradients: prediction by logistic regression. Marine Ecology Progress Series, Luhe, v. 225, p. 79-95, 2002.

ZAKLAN, S.; YDENBERG, R. The body size-burial depth relationship in the infaunal clam Mya arenaria. Journal of Experimental Marine Biology and Ecology, Amsterdam, v. 215, n. 1, p. 1-17, 1997.

ZWARTS, L.; WANINK, J. H. Siphon size and burying depth in deposit- and suspension feeding benthic bivalves. Marine Biology, Kiel, v. 100, p. 227-240, 1989. 